This isolated and severe $\gamma \mathrm{M}$ deficiency can be classified as a dysgammaglobulinaemia. It is distinct from the GiedionScheidegger variety (type 1), where $\gamma \mathrm{A}$ is also very low and where there is a continual history of all kinds of bacterial infections from birth. Case 4 initially showed a low $\gamma \mathrm{A}$ and $\gamma \mathrm{M}$, but this may have been due to delay in immunological maturation, for both are now developing normally. Cases 1 and 2 are also distinct from the Wiskott-Aldrich syndrome (type VA), a sex-linked recessive condition usually fatal to affected males before 10 years of age, where though $\gamma \mathrm{M}$ deficiency appears it is usually associated with a marked increase of $\gamma \mathrm{A}$. Clinically there is a long history of eczema, thrombocytopenia, and recurrent infections from soon after birth. Cases 1 and 2 have therefore been classified as type VB by Hobbs et al. (1967a). Furthermore, it is realized that isolated deficiency of $\gamma \mathrm{M}$ can be found in apparently healthy subjects (type VO) and can be associated with defects of other classes of immunoglobulins in both sexes of given families (Burtin et al., 1964). Thus the finding of $\gamma \mathrm{A}$ deficiency in four other relatives of Cases 1 and 2 adds support to a genetic basis for their fatal abnormality.

\section{Summary}

Serum immunoglobulin levels and meningococcal antibodies were measured in nine patients suffering from meningococcal infection. The bactericidal activity of normal serum to meningococci could be attributed largely to $\gamma \mathrm{M}$ globulin. Severe $\gamma \mathrm{M}$ deficiency, probably inherited, was found in two boys dying from fulminating meningococcal septicaemia. Other evidence supported the view that $\gamma \mathrm{M}$ antibodies are an important humoral defence mechanism and that the normal presence of these antibodies in the blood stream restrains systemic meningococcal infection to the C.S.F.

We thank Professor J. P. M. Tizard and Dr. J. A. Davis for allowing us to study patients under their care; Dr. S. Worlledge, who estimated the blood groups and isoantibody titres; and Professor L. P. Garrod for his advice. Requests for reprints should be addressed to Dr. J. R. Hobbs, Department of Chemical Pathology, the Royal Postgraduate Medical School, Ducane Road, London W.12.

\section{REFERENCES}

Adinolf, M., Glynn, A. A., Lindsay, M., and Milne, C. M. (1966). Immunology, 10, 517

Brown, J W. and Condit, P. K. (1965). Calif. Med., 102, 171. Burtin, P., Buffe, D., and Grabar, P. (1964). Ann. Inst. Pasteur, 106, 519.

Cohen, I. R., and Norins, L. C. (1966). Science, 152, 1257.

Evans, R. T., Spaeth, S., and Mergenhagen, S. E. (1966). F. Immunol., 97,112 .

Ewald, R. A., Williams, J. H., and Bowden, D. H. (1961). Vox Sang. (Basel), 6, 312 .

Goldman, J. M., and Hobbs, J. R. (1967). Immunology, 13, 421.

Heist, G. D., Solis-Cohen, S., and Solis-Cohen, M. (1922). 7. Immunol., $7,1$.

Hobbs, J. R., Russell, A., and Worlledge, S. M. (1967a). Clin. exp. Immunol., 2, 589 .

Immunol, 2, s89. A, and McSwiggan, D. A. (1967b). Lancet, 1 , 1108 .

Mattern, P., Masseyeff, R., Michel, R., and Peretti, P. (1961). Ann. Inst. Pasteur, 101, 382.

Michael, J. G., and Roven. F. S. (1963). F. exp. Med., 118, 619.

Muschel. L. H., and Jackson, J. E. (1966). F. Immunol., 97, 46.

Ross, C. A. C. (1961). Lancet, 2, 527.

- and Stevenson, J. (1962). F. Hyg. (Lond.), 60, 501.

Sample, D W Gauld, J. R., Warram, J. H. Winter, P. E., Runcik, K., and Go Gauld, (1966). Amer. I. Epidem. 84, 156.

Schultze, H. E., and Heremans, J. F. (1966). The Molecular Biology of Human Proletns, 1, 737 . London.

Skiörten F (1966). Acia pain microbiol. scand., 68, 517.

Skiörten. F. (1966). Acta pain microhiol. scand., 6 , Wong, R., and Good, R. A. (1966). J. exp. Med., 123, 615 .

\title{
Access to Circulation by Permanent Arteriovenous Fistula in Regular Dialysis Treatment*
}

\author{
J. S. HANSON, M.B., B.SC. ; M. CARMODY, M.B. ; B. KEOGH, M.B. ; W. F. O'DWYER, M.D.
}

Brit. med. F., 1967, 4, 586-589

Regular dialysis therapy has now become the accepted treatment of selected cases with end-stage renal disease.

Heretofore, repeated access to blood vessels for dialysis was achieved by means of the conventional Teflon or Teflon-Silastic prosthesis, establishing an external semipermanent arteriovenous shunt (Quinton et al., 1960, 1962 ; Hegstrom, 1961). In spite of its considerable success this technique is far from perfect, and problems of infections and clotting remain as a major cause of trouble in the management of these patients. The average survival rate for external shunts varies considerably from one centre to another. In a series published from the Swedish Hospital in Seattle the mean shunt life for both arteries and veins varied from 11 days to 11.4 months (Murray et al., 1964). In a more recent review from the same cenrre (Pendras and Smith, 1966) an average cannula survival of 12 to 14 months was reported. This they attributed to the prevention of infection in the patient before and shortly after operation, and also by avoiding cannulation in the severely uraemic state.

We have been unable to achieve a similar standard of shunt survival since we began regular dialysis therapy in November 1964. Our survival rates in arteries and veins were 3.8 and 3.6 months respectively, the majority of the failures occurring

- Department of Surgery and Renal Unit, Jervis Street Hospital, Dublin. in the first six weeks after surgery. The longest surviving shunt was 16 months. Infection and clotting, the major causes of our failures, were responsible for a high morbidity and often long periods of immobilization in hospital for surgical revision of the cannulas. This was particularly true for those in the lower limbs. The frequent manipulations involved in declotting were also a contributory factor to the psychological fixations which some of these patients developed about their cannulas. Our relative failure with the Quinton type shunt prompted us to adopt the surgically created arteriovenous fistula as described by Brescia et al. (1966). As a result of the arteriovenous fistula so created the arm veins are distended, allowing dialysis to be carried out by venepuncture. The veins are then in effect an extension of the arterial system.

\section{Surgical Technique}

Anaesthetic.-Brachial plexus block by the axillary route has been used routinely and has proved very satisfactory. Firstly, the accompanying sympathetic block dilates the blood vessels and prevents vascular spasm until the vein has been denervated by the stripping of its fascia. The sympathetic blockage may lead to free bleeding from the line of incision, but 
this can be controlled by circumvential infiltration around the wound with a local anaesthetic containing adrenaline. Secondly, the muscular paralysis enables the patient to lie in comfort with an outstretched arm for prolonged periods. Thirdly, the sensory effects of the brachial plexus block extends well into the postoperative period, and the analgesic effect is much appreciated by the patients. Fourthly, when performing this procedure under brachial block it is not necessary to admit the patient to hospital. Also it can be carried out at a time convenient to the patient and therefore does not interfere with his normal working hours.

\section{Choice of Vessels and Site of Fistula Formation}

In the early stages of this project we received patients who had had repeated shunt insertion. Frequently only ulnar arteries remained patent, the radial arteries having been cannulated. This does not make fistula formation impossible. Neither does it appear to impair seriously the blood supply to the hand if the sole remaining main vessel is used for fistula formation. In Tables I and II are given particulars of a series of 14 patients on regular dialysis treatment in whom an arteriovenous fistula was created surgically.

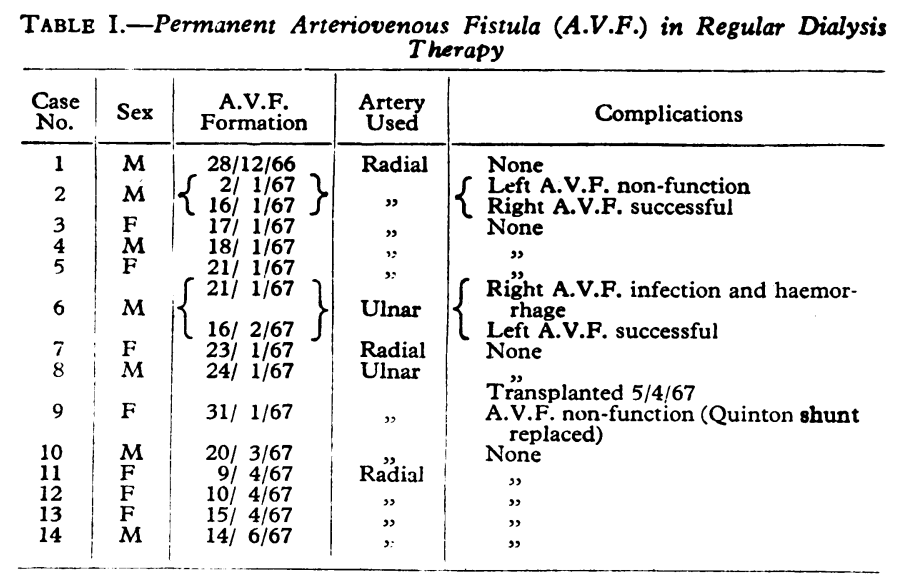

\section{TABLB II}

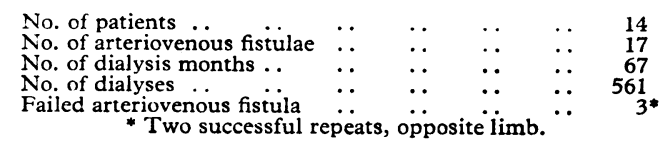

New patients introduced to the regular dialysis therapy programme cause no problem in respect of suitability of artery and vein, as they are initially fitted with a temporary Carmody (1964) tvpe shunt, leaving a virgin arm for fistula formation. When available the radial artery and cephalic vein are used routinely, but, as mentioned, the ulnar artery and any superficial vein in close proximity may be used. Venae comitantes do not give the flow required in the superficial veins. When using an ulnar artery it is preferable to avoid use of the small superficial veins in the area of the skin creases on the ventral carpal surface. In this situation the skin is tethered to the deep fascia, making dissection difficult and increasing the possibility of vessel spasm due to excessive handling. Again, the veins in this area form a branching network; therefore without ligating some branches it is unlikely that a segment of vessel adequate for fistula formation will be obtained. Finally, in association with brachial block there appears to be more superficial bleeding in the area.

In choosing a suitable vein a venous tourniquet is applied and the arm inspected. The availability of a suitable vein really dictates the site of fistula formation, but it is desirable to create it as far distally as possible in the arm to facilitate future venepuncture and tourniquet application. The depth of the artery from the surface does not militate against the use of a suitable vein.

A transverse incision is used routinely. Longitudinal incisions have to be of greater length to provide adequate exposure; also, they heal badly. The incision is made at the point of nearest proximity of artery and vein, unless the vein has an excessive number of branches in the area. A segment of vein without branches and sufficient for an anastomosis is necessary, as it is desirable to leave as many patent tributaries as possible. In the virgin arm the cephalic vein is constant in position and generally of good calibre, but in patients who have repeated shunt insertion and intravenous therapy most subcutaneous veins may be occluded. With an adequate cross anastomosis, veins that are quite minute enlarge and it seems that large thrombosed veins may recanalize. The skin incision should be of sufficient size to ensure that an adequate segment of vessel may be mobilized without tissue distortion due to retraction. Caution must be exercised to limit the incision to skin only, as otherwise the veins in the superficial fascia may be severed.

When the skin has been incised the superficial fascia is opened as far as the lateral margin of the vein on both sides. The skin and superficial fascia are undermined on both sides of the wound, leaving the vein encased in its own fascia. A venous tourniquet is applied to dilate the vein. The fascia on the vein is incised down to the adventitial layer at a central point and a sling is positioned here. From this point the vein is cleared by sharp dissection. Once the correct plane has been entered it is quite easy to clear the vein. To ensure maximum mobilization adjacent tributaries are cleaned in similar fashion. Another sling is now inserted and the vein is swung to the area of the artery, indicating the degree of mobility and adequacy of dissection. The vein lies in superficial fascia. In order to achieve approximation with the artery it must be slung deep to the fascia. This makes adequate undermining of the fascia a matter of paramount importance if "kinking" of the vein is to be avoided.

It must be emphasized that the completed arteriovenous fistula will be between deep and superficial fasciae, and "kinking " may occur at the junction of dissected and undissected segments of the vein. The tourniquet is now released, there being no danger of vessel spasm in the segment of vein to be used. The fascia over the artery is incised longitudinally. At one point the artery is freed from the venae comitantes and the sling inserted round the artery; the artery is dissected free. Only tributaries that will definitely interfere with its mobility are cut and tied. A second sling is inserted. With the use of both sets of slings, artery and vein are approximated (Fig. 1). The vessels must lie parallel for a distance of at least $2.5 \mathrm{~cm}$. between slings without tension or angulation if adequate fistula

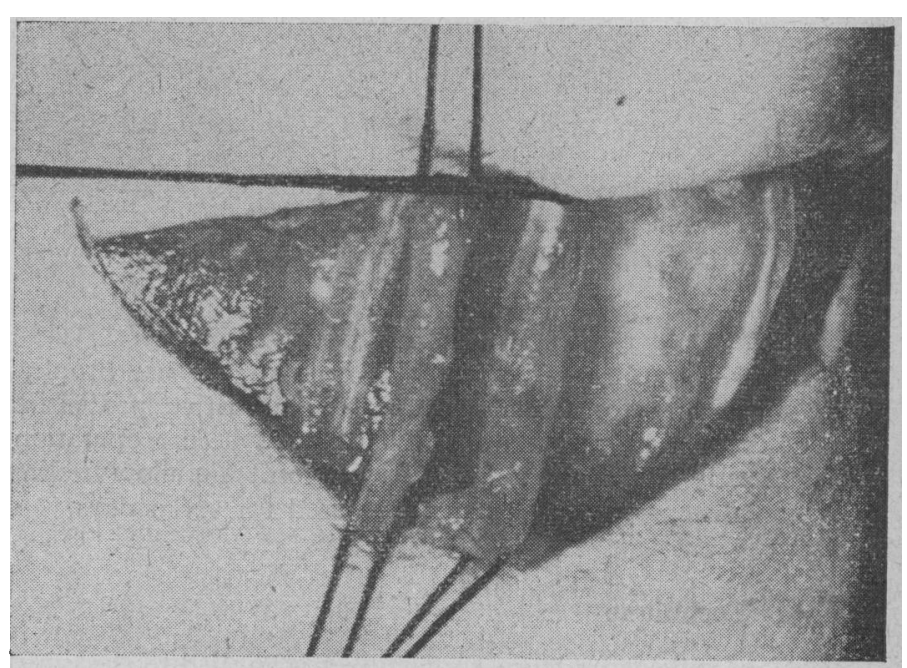

Fig. 1.-Vessels dissected and approximated. Use of slings indicating adequacy of dissection. 
formation is to be obtained. This is a critical point, as an equal flow in the vein, both proximally and distally, will otherwise not be obtained. Where the artery leaves and enters its own proper tissue plane an edge of fascia remains; this may "kink" the artery. It may therefore be necessary to extend the fascial incision proximally and distally. Both sets of slings are now replaced with De Bakey peripheral arterial clamps. These clamps are introduced from the assistant side. Longitudinal incisions $0.5 \mathrm{~cm}$. in length are made in corresponding lateral surfaces of vein and artery (Fig. 2). The lumen of both vessels is washed out with heparinized saline and a side-to-side anastomosis made in continuous fashion with 00000 polyethylene (Fig. 3). On release of the clamps there is an immediate bruit and thrill. The wound is sutured in two layers.

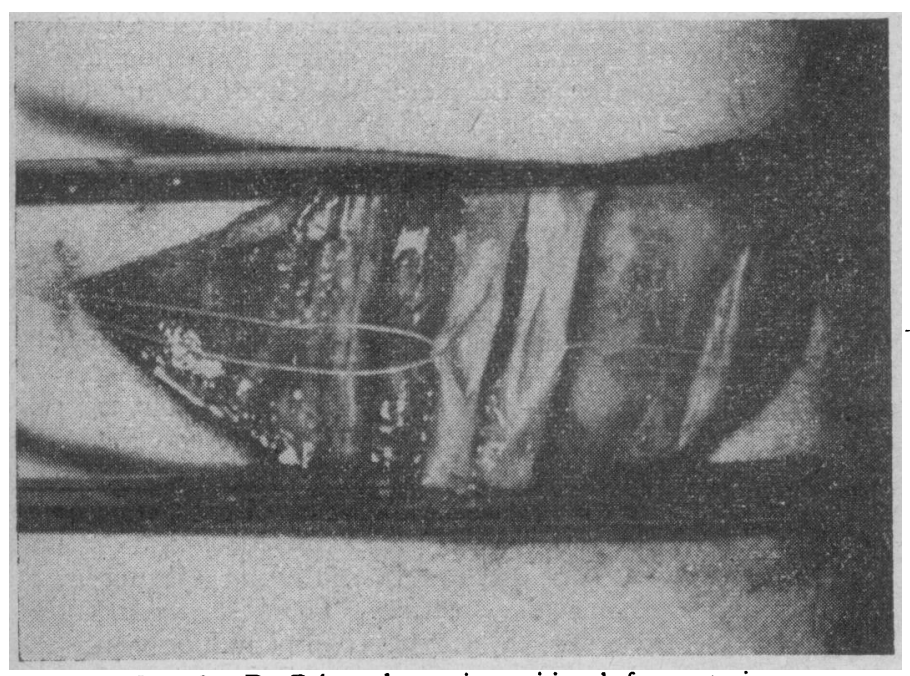

FIG. 2.-De Bakey ciamps in position before suturing.

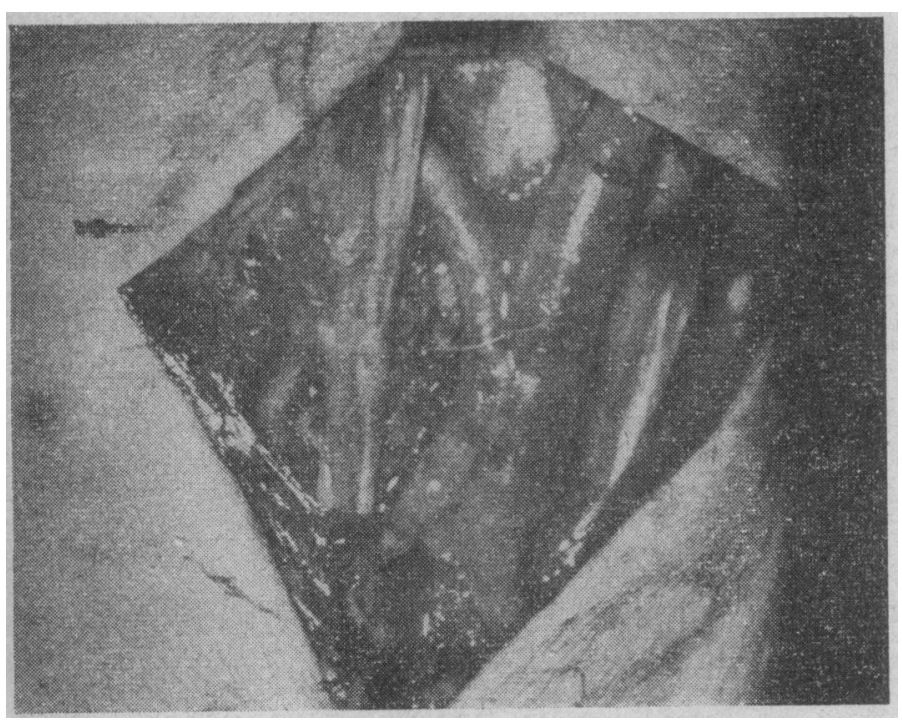

FIG. 3.-Arteriovenous fistula completed.

\section{Management}

Postoperative Care.-We feel it is necessary to use prophylactic antibiotics for five days after the operative procedure. It is essential that patients are well dialysed before operation, as this greatly lessens the hazard of infection. To allow healing and vein distension the fistula is not used for seven days.

\section{Dialysis Technique}

Previous to venepuncture a tourniquet is applied to the upper arm to obtain venous engorgement. The puncture sites are infiltrated subcutaneously with $0.5 \%$ lignocaine without adrenaline. Initially the obtaining of a suitable puncture needle was a major problem. We tried a variety of needles, but were continuously faced with the problem of clotting of the needles in the lumen despite increasing the standard dose of heparin by 10,000 units. However, we realized that the quality of our needles was at fault, the surface of the inside of the lumen being rough. We now satisfactorily use a $1 \frac{1}{2}$-in. $(3.8-\mathrm{cm}$.) 14 gauge thin-walled smooth-bore needle (Bardic). The needles are attached to Tomac extension tubes (Fig. 4). Firstly, the return-flow needle is inserted cephalically below the antecubital fossa and advanced maximally. The tourniquet is released and the standard dose of heparin is infused through this needle. Adequate time for heparinization is allowed. The tourniquet is now reapplied below the proximal needle and the outlet venepuncture is performed. If the needle is inserted proximal to the fistula it should be directed towards the hand, while if inserted distally it should point cephalically. The predialysis blood samples are taken from this latter tube.

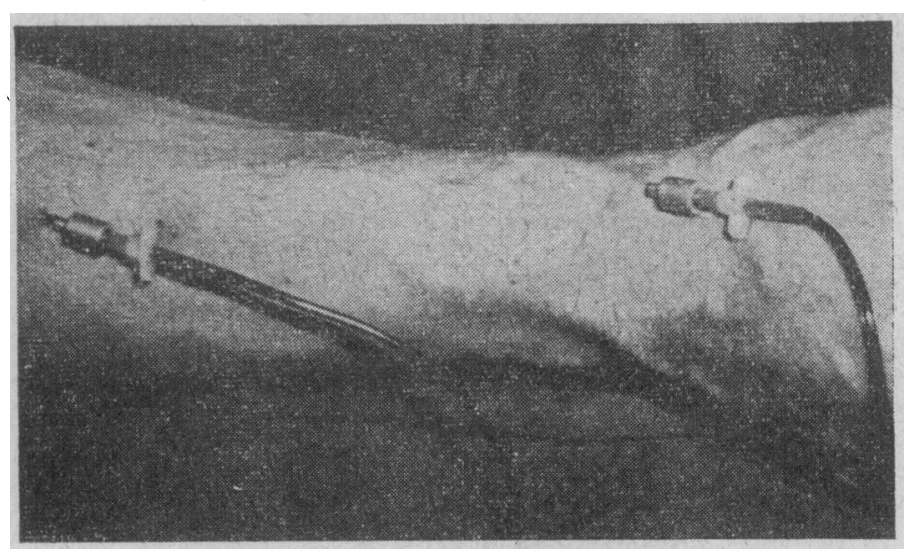

Fig. 4.-Needles in situ-dialysis in progress.

A light venous tourniquet is occasionally necessary between the needle insertions to ensure adequacy of flow. This is particularly so in the first few weeks after fistula formation, but later is unnecessary as the veins become distended and arterialized. Blood flow rates of $300 \mathrm{ml}$. per minute are easily obtainable by means of a roller type blood pump. Each dialysis lasts nine hours, and there is no falling off in the blood flow rates. At the completion of dialysis, protamine sulphate is infused. Each needle is removed at intervals of 10 minutes and digital pressure is applied by the patient along the line of venepuncture until haemostasis is obtained. This requires three to five minutes on an average. A light dressing is applied to the puncture sites, and the patient discards it after a few hours.

\section{Comment}

On our dialysis programme we use Kolff twin-coil machines, similar to those used by Brescia et al. (1966), who pioneered this technique of access to the circulation. In order to maintain an adequate flow rate with this technique some form of pump on the "arterial" side is necessary. Therefore the technique is not suitable for use on patients dialysed on a conventional Kiil type machine. In our experience with the external arteriovenous shunt (Quinton-Scribner) we must admit that our earlier regular dialysis therapy patients were inadequately dialysed owing to very demanding acute renal failure commitments. For the past eight months we have been able to correct this, and we recognize that inadequate frequency of dialysis may have increased our incidence of clotting and infection in the original external shunts. We are none the less impressed, however, by the Brescia-Cimino fistula, which allows much greater comfort and freedom for the patient. One woman on our programme has taken up golf since discarding her external shunt for the fistula. 
Since we adopted this method of access to the circulation for haemodialysis, the morale of each patient has changed considerably. Patients no longer live with their fears of an artificial Teflon shunt with its possible hazards and complications. They can rehabilitate themselves more easily and their lost working hours have decreased considerably. Similarly, life for members of the staff is no longer punctuated by frustrating crises of clotted shunts and infection.

We have not observed any evidence of cardiac embarrassment in spite of the alteration in circulatory dynamics produced by the introduction of the arteriovenous fistula.

We would like to suggest that this technique might be of value in circumstances other than haemodialysis where repeated access to the circulation is required-for example, repeated transfusions in aplastic anaemia. In this regard Williams et al. (1967) and Anderson et al. (1967) have reported on the successful use of the conventional type of Quinton-Scribner Teflon shunt in a total of three patients with aplastic anaemia requiring regular transfusions. It is noted, however, that in two cases the problem of clotting occurred. From our experience we would suggest that a permanent surgically created arteriovenous fistula as described here would be a less troublesome method of obtaining access to the circulation when repeated transfusions are required.

\section{Summary}

An arteriovenous fistula was created surgically in 14 patients on regular dialysis treatment in order to provide repeated access to the circulation. In 11 patients the initial arteriovenous fistula functioned satisfactorily and without complica- tions. In two patients in whom the initial fistula failed to function, success was achieved in the opposite forearm. In one patient in whom the procedure failed initially a second attempt to create a fistula has been postponed owing to the poor calibre of her veins and the urgency of maintaining adequate haemodialysis.

ADDENDUM.-Since this communication was submitted for publication two further patients have had successful arteriovenous fistulas established. In addition one patient (Case 9) in whom arteriovenous fistula formation on 31 January 1967 was unsuccessful and in whom a Quinton shunt was replaced has recently had a functioning fistula established by use of her remaining ulnar artery. In September 1967 the total number of patients with successful fistulas was therefore 16 .

\section{REFERENCES}

Anderson, D. C., Emslie-Smith, D., Macgregor, A. M. C., and Stewart, W. K. (1967). Lancet, 1, 509.

Brescia, M. J., Cimino, J. E., Appel, K., and Hurwich, B. J. (1966). New Engl, 7 Med, 275, 1089 .

Carmody, M. (1964). Proceedings of European Dialysis and Transplant Association, No. 1.

Hegstrom, R. M., Quinton, W. E., Dillard, D. H., Cole, J. J., and Scribner, B. H. (1961). Trans. Amer. Soc. artif. intern. Organs., 7, 47.

Murray, J. S., Pendras, J. P., Lindholm, D. D., and Erickson, R. V. (1964). İbid., 10, 191 .

Pendras, J. P., and Smith, M. P. (1966). Ibid., 12, 222.

Quinton, W. E., Dillard, D. H., Cole, J. J., and Scribner, B. H. (1962). Ibid., 8, 236 .

Ibid., 8, 236.

Williams, B. T., Blainey, J. D., Dawson-Edwards, P., Hilton, D. D., and Simpson, K. M. (1967). Brit. med. F., 2, 484.
- Lecturer in Pharmacology and Therapeutics, University and Royal Infirmary, Sheffield.

t Formerly Boots Research Fellow, Department of Pharmacology and Therapeutics, University and Royal Infirmary, Sheffield.

\section{Patients and Methods}

Subtotal Thyroidectomy Group.-Patients received intravenous calcium infusions two or three days before and at the same interval after subtotal thyroidectomy for thyrotoxicosis (five) or non-toxic goitre (four); thus each patient acted as his own control. Antithyroid drugs and potassium iodide were given preoperatively to patients with thyrotoxicosis.

Radioiodine Therapy Group.-Our studies included 24 patients who had been treated with radioiodine for thyrotoxicosis from 2 to 15 years previously and a group of seven patients of similar age without any evidence of thyroid disorder who agreed to help in this investigation after its value had been fully explained to them.

Technique.-The intravenous infusion of calcium was given at a constant rate over four hours by means of a D.C.L. micropump type T (Messrs. F. A. Hughes \& Co. Limited). The dose of elemental calcium was $15 \mathrm{mg} . / \mathrm{kg}$. body weight given as a calcium gluconate solution (15 mg. of calcium per $5 \mathrm{ml}$.). Blood samples were taken before ( 0 hours) and immediately after the infusion (4 hours), and subsequently at 8,12 , and 24 hours. The serum calcium was estimated by an edetic acid titration with calcichrome as indicator and a manual titrator (Messrs. Evans Electroselenium Ltd.). The rise in serum calcium and elevation above pre-infusion level was plotted at four- 